\title{
Development of Smart Multiplatform Game App using UNITY3D Engine for CPR Education
}

\author{
Ji Won Oak ${ }^{1}$ and Jae Hwan $\mathrm{Bae}^{2 *}$ \\ 1 Department of Nursing, Tongmyong University, Busan, South Korea \\ jiwonoak@gmail.com \\ 2 Department of Game Engineering, Tongmyong University, Busan, South Korea \\ bjhmail@tu.ac.kr, Corresponding author \\ Abstract
}

The purpose of this study was to spread CPR education to the public and to promote the effects of training using an app design that applied the gamification theory based on UNITY3D engine. The results of this study are expected to contribute to the advancement of the online smart SNG market through the promotion of the net funchonal effects of games by applying the gamification theory on various functional games, and by using UNITY3D to convert web-based games and contents into IOS' or 'ANDROID' and widely developing them into smartphone-use services. Alsothe clients incommunity would likely be equipped with an ability to respond to emergency situations. by receiving CPR training in a fun and easy manner at anytime, anywhere

Keywords: CPR(Cardiopulmonary resuscîtation), Social network games, Gamification, Smart multiplatform games

\section{Introduction}

Cardiopulmonary resuscitation (CPR) is an emergency procedure, performed in an effort to manually preserve intact brain function until further measures are taken to restore spontaneous blood Cireulation and breathing in a person in cardiac arrest [1].

Cardiac arrests occur suddenly and the patients are not usually able to call for assistance on their ow 0, 4-5 minutes after a cardiac arrest brain damage starts to take place; therefore, a quick first aid action by a witness is essential [2-3]. With each minute of delay indefibrillation the survival rate can decrease by $7 \%-10 \%$, however, this can be reduced by $2.5 \%-5 \%$ per minute if a cardiopulmonary resuscitation (CPR) is performed. In cases where a witness performs a CPR, the survival rate can be 2-3 times higher [4]. The rate of CPRs being performed by the general public in South Korea is 3.1\% Which is very low when compared to those of Sweden at $77 \%$, the United States $37.4 \%$, and Japan at $12.0 \%$ [5]. The rate at which a patient suffering from an acute cardiac arrest survives the transport and is admitted to the hospital in Korea is $9.9 \%$ and the rate for a live discharge from hospital is only $3.0 \%$; moreover, in particular, the rate for a normal brain function is a mere $0.9 \%$. Whereas, in the case of the United States, the rates are $26.4 \%$ for a live admission to the hospital, $10.3 \%$ for a live discharge, and $7.7 \%$ for a normal brain function. In other words, whether or not a CPR is performed by a witness on a patient suffering a cardiac arrest occurring outside the hospital becomes an important factor that determines the patient's prognosis [6]. Currently, in the United States and Europe, the encouragement and support for CPR and first aid training for the general public are actively being pursued [6]. 
In 2008, in Korea as well, a legislation for the installation and use of automated external defibrillators (AED) was put in place, and since 2010, how-to-use training has been underway and CPR training is being conducted through the health care training in schools [7]. However, due to the fact that CPR training is optional and not mandatory and due to other factors, including the lack of professional teachers, inadequate training materials and facilities, the lack of interests, etc., there exists a problem of CPR training for the general public not being expanded sufficiently [4, 8-9]. In addition, since CPR entails a layperson performing an unfamiliar medical procedure in an emergency situation, what is required in CPR training is not just a simple transfer of knowledge and skills but also a repetitive training that instills self-confidence and self-ássurance about one's own ability while overcoming the psychological-social stress factors that accompany performing CPRs. Therefore, this study proposes the following: the current training infrastructure, which is insufficient for spreading CPR training for the general public, needs to be complemented; publicity and interests heed to be strengthened; and a CPR training method that ensures confidence and assurance needs to be devised.

SNG is a game that's rapidly growing together with the popularization of smartphones on the SNS level, and it is a service that fuses the advantages of SNS such as Twitter, blogs, Facebook, etc. with the enjoyment of games [6]. The unique feature of SNG is that it is a new communication means that ditizes the social relationships built in SN where networking can be achieved while enjoying games with a number of gamers, and it is making advances white showing apotential for the game space to be expanded into the social space. It can be saje that it's an effective form of game, in which, by evolving from the existing games that emphasize personal accomplishments and rewards, the interest leven is increased because of the interaction among social activity players becomes an important etement of game playing, and participation by the many is induced. Such an SNG can be applied for CPR training in that, by combining a variety of realistic materids such as texts usable in smartphones, videos, sounds, etc., and it can provide data and assistance in promoting correct understanding of CPR by the general public [6]. Furthermore, it is expected that the games that are associated with text service and SNS service of the smartphones will promote interaction among the applicable subjects and provide increased interests and publicity benefits for CPR training [6].

"Gamification" is utilized as a process that solves problems through game-like thinking and game mechanics while facilitating participation by the users. In gamification, through the problem-solving activity of game stories with a play-minded approach and through fun, etc., voluntary participation by the users is encouraged and immersion is enabled [6]. This increases problem-solving skills through a step-by-step leaning and, through the functional characteristics of games that enable achieving a clear sense of purpose of rules and strategies, induces the subjects to solve problems on their own and brings out their participation and positive behavior. The strong points of gamification, which clearly identify the purpose, tasks, behaviors, etc. that need to be achieved and which provide rewards when these are achieved, will facilitate the subjects to recognize and deal with the situations that they are facing in the culture where individuals are living, and the elements of fun, scores, level-ups and ranking competitions are deemed to be able to play the role of sustaining voluntary participation by inducing the internal and external motivations of the subjects [10]. Therefore, the application of the gamification theory on CPR games is expected to use an important factor that sustains the effects of training and participation. 


\section{Development of Multiplatforms that Use UNITY3D Engine}

In order to produce multiplatform games, for each platform, all the different settings of the system must be controllable. In this paper, the use of Unity3D engine is proposed based on the reason that codes can be written with C\#, JavaScript and Boo, the Unity3D engine was developed as a C\#- and Mono-based code. The run-time part of the engine was developed with $\mathrm{C}++$ and Microsoft NET API, and the editor program was developed with C\#. As for the script, it cannot be modified directly in Unity, and it can be modified in script editors, such as Mono Develop, etc., that support Unity. Although Visual Studio also supports through plug-ins, it lacks overall debugging functionality and, in the Express version, debugging is not supported. In the case of Unity3, not only simple physics engines or shaders, but also realistically, all conditions, including network or terrain manipulation, audio, video, animations, etc., are supported. By converting web-based games and contents developed using Unity $3 \mathrm{D}$ into 'IOS' or 'ANDROID,' they can be serviced as smartphone-use (Figure 1) [10-11].)
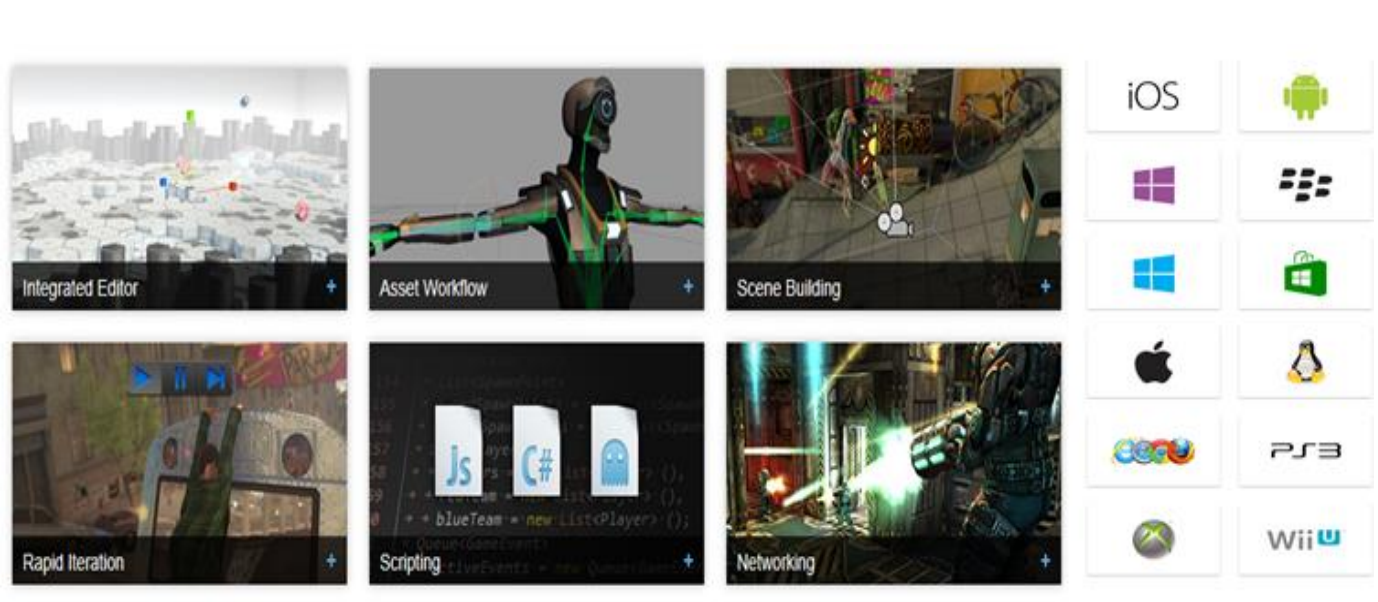

Unity3Dsupports 3cype of script languages: Java Script, C\# and Boo. All three are capable of the same fast interaction, capable of utilizing .NET libraries, and all three support databases, standard expressions, XML, networking, etc. Even though scripting is normally considered to be limited and slow, Unity3 operates with very fast iteration times and, by pairing up with a compilation of easy native code, becomes almost as fast as the $\mathrm{C}+$ level. In addition, the implementation of Java Script for Unity3D operates at the same speed as C\# or Boo. Game logic is executed in the .NET platform Mono open source: which provides a best-in-the-world programming environment that offers the best speed and flexibility [6].

A single line of code is sufficient for moving, rotating and scaling an object. The replication, removal and changing of the properties are, of course, the same. Everything can be directly referenced via the names, layers, tags, similarity, or touch, etc. The functionality of Unity3D is integrated with all the MonoDevelop, in license. As shown in Figure 2, this would mean that Unity3D would have IDE of the highest level [6].

Unity3D is automatically synchronized with MonoDevelop and as well as with Visual Studio. All internal references are set so as to attain a complete auto-completion in the internal API. Unity3D is capable of browser communication with Web Player autonomously. The HTML page containing Unity Web Player contents are able to communicate with the 
contents and also the vice versa is possible. Basically there are two communication directions. When importing the profile information of individuals through the OpenSocial API from a site that offers SNG, communication between the browser and web player, and in Unity, issues can easily be resolved by using the GetUnity(). SendMessage function and the Application. ExtenalCall function. This can be deemed essential in creating a social network game [6].

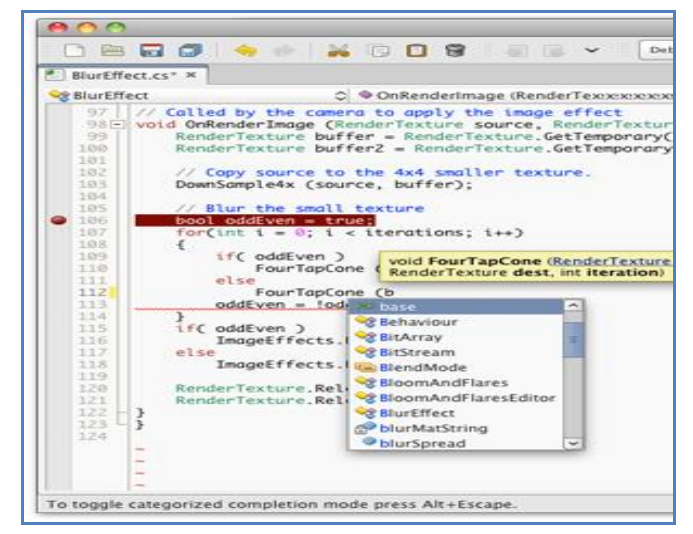

Figure 2. Unity3D Mono Development

\section{SNG Programming Tecmique .}

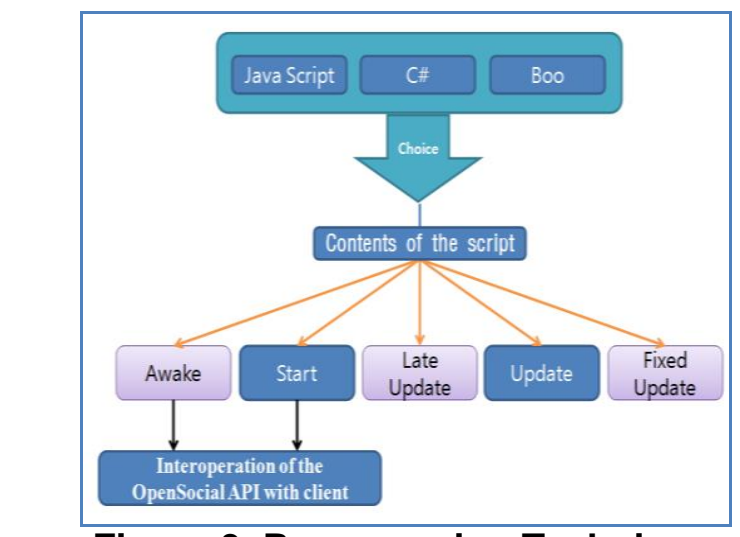

Figure 3.Programming Technique \&

Scripting in Unity supporß all three Panguages of Java Script, C\# and Boo; in addition, all 3 languages can be used in one project. As such, without any restriction of one language, script can be written freely. However, within a script, only one script language can be used.

The first thing one needs to know yyen writing a script in Unity is a Reference for which Overriding is possible; in whieh, the most important are the Update, LateUpdate, FixedUpdate, Awakeand Start The Updatefunction is called for every frame when the script is on. As the most frequently-used function in the script, the Time.deltaTime can be used for finding out about the pass time. The LateUpdatefunction is also called for every frame, and it is called after all the Updatefunctions have been called. The FixedUpdatefunction, too, is called for exery frame, and it is used in lieu of the Updatefunction when treating the Rigibody. The Awakefunction is called when a script object is being loaded. It is used to initialize variables or conditions before the game begins, and it is called only once during the lifetime of a script object. Because the Awakefunction is called after all the objects have been initialized, it can use safely all the functions likethe GameObject.FindWithTag. When the Updatefunction is called the first time, the Startfunction is called right before the Updatefunction. It is called only once during the lifetime of a script object, and the Awakefunction is always called right before the Startfunction [6].

\section{CPR Game Content Design}

This game was developed for the prevention of deaths of the large number of cardiac arrest suffering patients due to not receiving a CPR within the appropriate time [6].

The game is a smart SNG based on multiplatforms all applicable in the iPhones, Android phones, Windows Mobile and the PC. The game players discover a variety of cardiac arrest patients, such as accidental drowning patients vacationing with the family, fainted hiker 
patients on the mountain, unconscious patients from traffic accidents, etc. The play that discovers a patient calls 911 and then, by performing a CPR, the player keeps the cardiac arrest patient alive until the ambulance arrives. In actuality, the players perform artificial respiration and chest compressions by using the touch pad and, by using SNS through SNG connections, they can compete with many other players (Figure 4). This game's simple interface, short game time of about 1-2 minutes, and the introduction of a score competing system maintain the interest level of the game; which, in turn, facilitate the local community members to learn how to perform CPR per the correct method and save lives and prevent complications when they run into cardiac arrest patients in various settings. Also, this game provides an opportunity to check the locations of nearby defibrillators and to learn the proper method of use, and the game offers an advantage of promoting the importance of CPR by spreading the game via SNS [6].

\section{Conclusion}

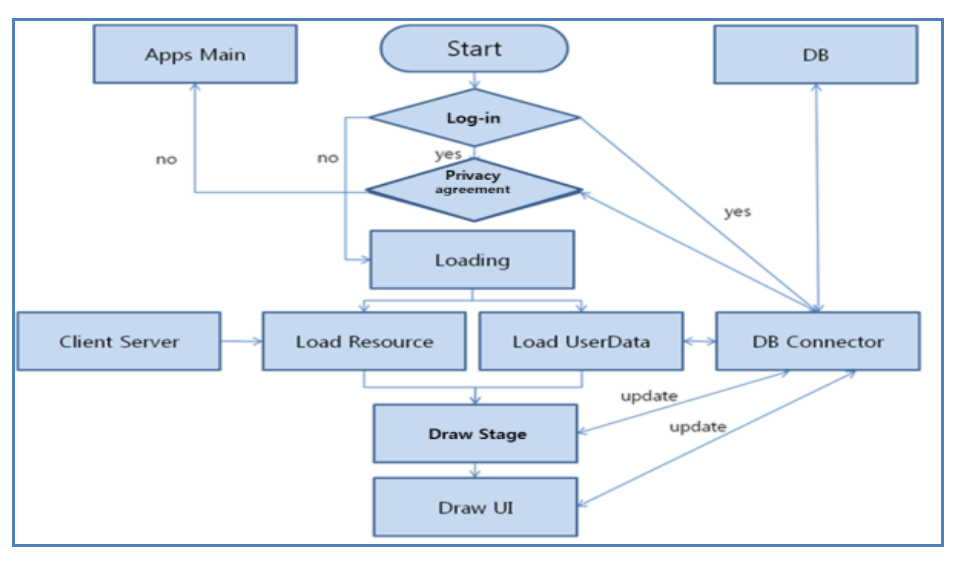

Figure 4.'CPR game; flow chart

This study proposed to proyide CPR training to the general public and to promote the effects of training. An appy design was proposed that applied the gamification theory based on smart multipatforms using UNITY3D engine. The results of this study are expected to contribate to the advancement of the online smart SNG market through the promotion of the net functional effects of games by applying the gamification theory on various functional games, and by using UNITY3D to convert web-based games and contents into 'YOS' or 'ANDROID' and widely developing them into smartphone-use services [6]. Also, the clients in community would likely be equipped with an ability to respond to emergency situations, by receiving CPR training in a fun and easy manner at anytime, anywhere.

\section{References}

[1] J. M. Field, M. F. Hazinski and M. R. Sayre, et al "Part 1: executive summary: 2010 American Heart Association Guidelines for Cardiopulmonary Resuscitation and Emergency Cardiovascular Care”, Cir, vol. 122, (18 Suppl 3), (2010), pp. S640-656.

[2] M. J. Lee, "Incidence and outcome of cardiac arrest in Korea", J. Korean Soc. Emerg. Med, vol. 23, (2012), pp. $168-180$.

[3] S. O. Hwang, M. E. Ahn, Y. S. Kim, K. S. Lim, J. H. Yun and K. H. Choe, "Outcome of resuscitation victims of prehospital cardiacarrest”, J. Korean Soc. Emerg. Med., vol. 3, (1992), pp. 27-36.

[4] S. O. Hwang and K. S. Leem, "Cardiopulmonary Resuscitation and Advanced Cardiovascular Life Support", Koonja publishing, Seoul, (2011). 
[5] B. C. Lee, M. J. Lee, S. J. Shin, H. O. Ryu, J. K. Kim, J. B. Park and K. S. Seo, "The Current Status of Cardiopulmonary Resuscitation Training for School”, J. Korean Soc. Emerg. Med., vol. 23, no. 4, (2012), pp. 470-478.

[6] J. W. Oak and J. H. Bae, "Current Research on Game and Graphics", Proceedings International Workshop, Game and Graphics 2013, (2013) December 11-13, Jeju Island, Korea.

[7] H. S. Kim, D. C. Uhm and S. W. Hong, "Factors Influencing Cardiopulmonary Resuscitation Performance on a Stranger", J. Korean Acad. Soc. Nurs. Edu., vol.16, no. 2, (2010), pp. 339-346.

[8] C. A. Lester, C. F. M. Weston, P. D. Donnelly, D. Assar and M. J. Morgan, "The need for wider dissemination of CPR skills: are schools the answer?", Resuscitation, vol. 28, (1994), pp. 233-237.

[9] A. Mills, E. Wilson and W. A. Tweed, "Heart-alert; evaluation of a community training program for cardiopulmonary resuscitation”, Can Med Assoc. J., vol. 124, (1981), pp. 1135-1139.

[10] J. H. Shin "Utilizing Mobile Social Game as a Gamification Advertising Platform", J. of Contents Association, vol. 13, no. 4, (2013), pp. 86-96.

[11] Unity Korea, "Unity Korea", http://unity3dkorea.com

[12] UNITY: Game Development Tool, "UNITY", http://unity3d.com/unity/engine/programming,

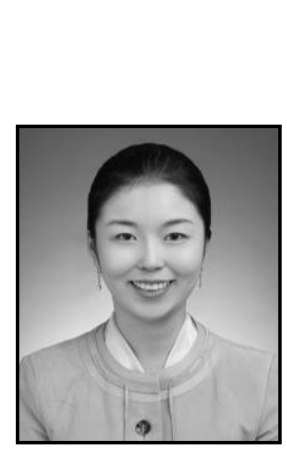

\section{Authors}

Ji Won Oak, She received her Ph.D. degrees in 2004 and 2007 from Dept. of Nursing of Ewha-Womans University in Seoul, Korea. She is currently an assistant professor/with the department of Nursing, Thongmyong Oniversity. She is research interests are in Nursing Education and prevention program.
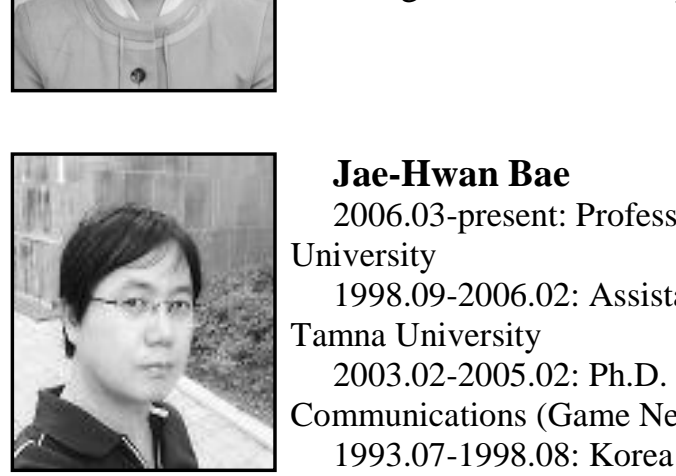

Jae-Hwan Bae

2006 03 present: Professor, Department of Game Engineering, Tongmyong University

1998.09-2006.02: Assistant Professor, Department of Computer Games,

\section{Tamna University}

2003.02-2005.02: Ph.D. Daegu University (Major: Computer and

Communications (Game Network))

1993.07-1998.08: Korea Data Communication Co., Ltd.

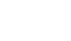

\section{8}

